\title{
Indirect (and direct) dark matter searches at the ICRC 2019
}

\section{Christoph Weniger*}

GRAPPA, Institute of Physics, University of Amsterdam

E-mail: c.weniger@uva.nl

The nature of dark matter remains one of the most important outstanding problems of astroparticle physics and cosmology. Progress on indirect and direct searches for non-gravitational signals from dark matter has been reported in over 50 contributions to the 36th International Cosmic-Ray Conference in Madison. Besides updates on well-established searches for Weakly Interacting Massive Particle dark matter, which continue to improve in sensitivity by 1-2 orders of magnitude per decade, a wide range of new/alternative dark matter search strategies were discussed. These include new detection strategies, new targets, and searches for, e.g., hidden photons, axion-like particles, primordial black holes and strange quark nuggets. Here, I provide a succinct overview of these contributions, including expectations for the upcoming years.

36th International Cosmic Ray Conference -ICRC2019-

July 24th - August 1st, 2019

Madison, WI, U.S.A.

${ }^{*}$ Speaker. 


\section{Introduction}

The nature of dark matter (DM) in the Universe remains an open problem, and constitutes one of the most exciting and complex questions of astroparticle physics and cosmology today. Since the quest for DM took off in the 1980s, dark matter research is a highly active field. The dark matter contributions to the 36th International Cosmic Ray Conference in Madison provided a good cross-section of recent activities, with strong focus on indirect detection methods. This included updates on well established methods for WIMP (Weakly Interacting Massive Particles) searches, as well as an increasing number of contributions that covered searches for non-WIMP signals, including secluded DM with long-lived mediators, axion-like particles, primordial black holes, hidden photons and nuclearites. Also updates on direct searches, in particular related to probes of the DAMA signal, were presented. I will here provide an overview of all contributions, and highlight the (from my perspective) most notable results. Due to the page limit, I will limit the references exclusively to conference contributions; all further references can be found therein.

The document is organized as follows: In Sec. 2, I discuss DM searches with photons, including classical WIMP searches as well as, e.g., searches for primordial black holes or nuclearites. In Sec. 3, I will discuss searches using cosmic-ray neutrinos. Dark matter searches with positrons and anti-nucleons are discussed in Sec. 4 and Sec. 5, respectively. Laboratory experiments, including direct WIMP searches as well as hidden photon searches, are discussed in Sec. 6. I will summarize and conclude in Sec. 7.

\section{Dark matter searches with photons}

Dark matter annihilation or decay into Standard Model particles produces usually a host of photons. In the case of hadronic final states (often assumed to be $\chi \chi \rightarrow \bar{b} b$ ), these photons stem mostly from the decay of neutral pions produced during hadronization; in the case of leptonic final states, final state radiation can play an important role, and to somewhat to significantly harder spectra. These mechanisms give rise to characteristic energy spectra, which are searched for in gamma-ray observations. In some scenarios (e.g., Wino DM), higher-order corrections can lead to more pronounced features and enhanced signal intensity.

\subsection{WIMP searches in dwarf galaxies and subhalos}

Dwarf spheroidal galaxies (dSphs) are excellent targets for DM searches, due to their large mass-to-light ratio, and the fact that their DM-dominated mass can be well constrained via observations of their member stars kinematics. On top of the eight classical dwarf galaxies, many more have been found during the recent years, mostly thanks to the Dark Energy Survey (DES). An update of searches for ultra-faint dSphs using three years of DES data was presented in Ref. [1]. However, no new dSph were identified.

Ref. [2] presented preliminary results on dark matter searches in $20 \mathrm{dSphs}$ that combine experimental results from the IACTs HESS, MAGIC and Veritas, as well as the water Cherenkov array HAWC and the satellite telescope Fermi-LAT. Due to the different location and energy range of each instrument, these observations are complementary. 
The Fermi Large Area Telescope (LAT) is operating since 2008. It is a pair conversion detector, using a silicon strip tracker for positional and cesium iodine calorimeter for energy reconstruction. Fermi-LAT is sensitive to gamma-ray energies down to $20 \mathrm{MeV}$, and up to above $300 \mathrm{GeV}$, and has with $20 \%$ a very large field of view. Most of the time Fermi is operating in a survey mode that covers the full sky every 3 hours. The HESS, MAGIC and Veritas observatories are Imaging Atmospheric Cherenkov Telescopes (IACTs). High-energy gamma-rays produce a shower of secondary particles when interacting with Earth's atmosphere, generating Cherenkov light that provides information about the direction and energy of the initial gamma ray. The five IACTs of HESS are situated in the Southern Hemisphere in Namibia at a height of $1800 \mathrm{~m}$. The initial HESS I instrument with four $12 \mathrm{~m}$ (diameter) dishes was recently extended by HESS II, a single $28 \mathrm{~m}$ dish, relevant for low gamma-ray energies. HESS I and II cover now the energy range from $30 \mathrm{GeV}$ to $100 \mathrm{TeV}$. The MAGIC observatory has two IACTs with $17 \mathrm{~m}$ diameter that were built in 2004 and 2009. It is situated in the Northern hemisphere, at an altitude of $2200 \mathrm{~m}$ on La Palma. Its energy range covers tens of $\mathrm{GeV}$ to several hundred of $\mathrm{TeV}$. The four $12 \mathrm{~m}$ dishes of Veritas were build between 2004 and 2007, in the Northern hemisphere, Arizona, at an altitude of $1268 \mathrm{~m}$. The energy range covers $100 \mathrm{GeV}$ to $30 \mathrm{TeV}$. Finally, the High Altitude Water Cherenkov (HAWC) gamma-ray observatory is built in Sierra Nevada at an altitude of $4100 \mathrm{~m}$. It consists of 300 Water Cherenkov detectors, that measure charged particles produced in atmospheric showers that are induced by high-energy gamma rays. The instrument is sensitive to gamma rays in the range $300 \mathrm{GeV}$ to $100 \mathrm{TeV}$. A distinguishing feature w.r.t. IACTs is with $15 \%$ sky coverage at any moment the very large field of view. An overview of different DM searches activities with the HAWC detector was presented in Ref. [3].

In Ref. [2], multiple dSphs were included from each of the five experiments, in a combined likelihood approach to bring together all data. The analysis allows to consistently treat uncertainties in for instance the DM content of the dSphs. No significant gamma-ray excess was found after combination of all results. In Fig. 1, the preliminary constraints on the annihilation cross-section into $\bar{b} b$ final states are shown [2]. The results are stronger than previous constraints, showing the usefulness of combining individual constraints. DM masses below $m_{\chi} \approx 100 \mathrm{GeV}$ are excluded for $s$-wave annihilation. At these DM masses, the constraints are dominated by the Fermi-LAT experiment. Interestingly, they are significantly stronger than previous Fermi-LAT results [4], which were based on a much larger number of dSphs (39), but which only excluded DM matter masses below $70 \mathrm{GeV}$. This effect is presumably caused by the absence of a few targets with upward fluctuations (like Ret II) from the list shown in Fig. 1.

Results from 80 h of HESS searches (conducted in 2017/2018) for DM signals from several nearby ultra-faint dSphs found by DES were presented in Ref. [5]. No significant excesses were found at $\gtrsim 100 \mathrm{GeV}$ energies, and the strongest constraints reach down to $(\sigma v)_{0} \sim 10^{-24} \mathrm{~cm}^{3} \mathrm{~s}^{-1}$ cross-sections for $m_{\chi} \sim 1 \mathrm{TeV}$ (depending on the final state). Furthermore, results from HAWC searches for DM signals from dwarf Irregular galaxies were presented in Ref. [6]. Dwarf irregular galaxies (dIrr, potentially predecessors of dSphs) are dominated by DM and have a low star formation rate, which makes them good targets for DM searches. The analysis was based on 31 dIrr galaxies in the HAWC field-of-view. No excess was found, and the upper limits reach down to $(\sigma v)_{0} \sim 10^{-24} \mathrm{~cm}^{3} \mathrm{~s}-1$, depending on the final state, covering DM masses between 1 and $100 \mathrm{TeV}$. 
Finally, HESS searches for DM signals from the dIrr galaxy WLM (Wolf-Lundmark-Melotte; also included in the HAWC analysis) were presented in Ref. [7], based on 19 hours of observations in 2018. No signal was found. The obtained constraints remain a few orders of magnitude weaker than the constraints obtained by HAWC, presumably because of the much shorter effective observation time.

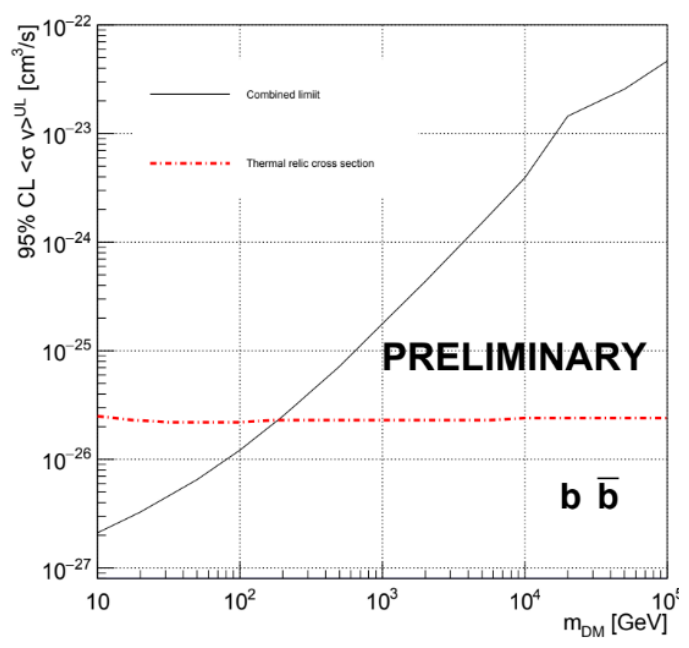

\begin{tabular}{l|l}
\hline \hline Source name & Experiments \\
\hline Boötes I & HAWC, VERITAS, Fermi-LAT \\
Canes Venatici I & Fermi-LAT \\
Canes Venatici II & Fermi-LAT, HAWC \\
Carina & HESS, Fermi-LAT \\
Coma Berenices & HAWC, HESS, Fermi-LAT \\
Draco & HAWC, Fermi-LAT \\
Fornax & H.E.S.S., Fermi-LAT \\
Hercules & HAWC, Fermi-LAT \\
Leo I & HAWC, Fermi-LAT \\
Leo II & HAWC, Fermi-LAT \\
Leo IV & HAWC, Fermi-LAT \\
Leo T & Fermi-LAT \\
Leo V & Fermi-LAT \\
Sculptor & H.E.S.S., Fermi-LAT \\
Segue I & MAGIC, VERITAS, HAWC, Fermi-LAT \\
Segue II & Fermi-LAT \\
Sextans & HAWC, Fermi-LAT \\
Ursa Major I & HAWC, Fermi-LAT \\
Ursa Major II & HAWC, MAGIC, Fermi-LAT \\
Ursa Minor & Fermi-LAT \\
\hline
\end{tabular}

Figure 1: Left panel: Combined constraints on the dark matter annihilation cross-section into $\bar{b} b$ final states, based on HESS, HAWC, MAGIC, Veritas and Fermi data. Right panel: List of dSphs included in the analysis. From Ref. [2].

In Ref. [9], the authors studied with HESS several candidates for DM subhalos, in the form of Fermi-LAT unidentified sources with very hard spectra. The selection criterion was that the sources, if interpreted in terms of DM, would be likely observable also with HESS. However, none of the sources was detected with HESS, which was used to constrain the DM mass (assuming that the observed objects are indeed indication DM annihilation signals from subhalos of the Milky Way).

In the future, the Large High Altitude Air Shower Observatory (LHAASO) is likely to provide strong constraints on very heavy DM annihilation, for DM masses around 1-100 TeV, that can obtained from observations of dSph galaxies. LHAASO is a next generation hybrid cosmicray/gamma-ray instrument that is going to be build at $4410 \mathrm{~m}$ altitude in the Norther hemisphere, the Sichuan province of China. It combines water Cherenkov and Cherenkov telescope techniques, which allows to cover a wide field of view ( $\sim 2 \mathrm{sr}$ ) and gamma-ray energies between $300 \mathrm{GeV}$ and $1 \mathrm{PeV}$ [8]. Sensitivity estimates, based on 1 year of raw observation time, were presented in Ref. [8], and are here reproduced in Fig. 2. These constraints are based on a combined analysis of $19 \mathrm{dSphs}$, and dominated by Segue I, Ursa Major II and Triangulum II. Although these future constraints are still about two orders of magnitude above the thermal cross-section, they have the potential to improve on current dSph HESS, Veritas and MAGIC results by up to a factor of five, in particular for DM masses above $10 \mathrm{TeV}$. 

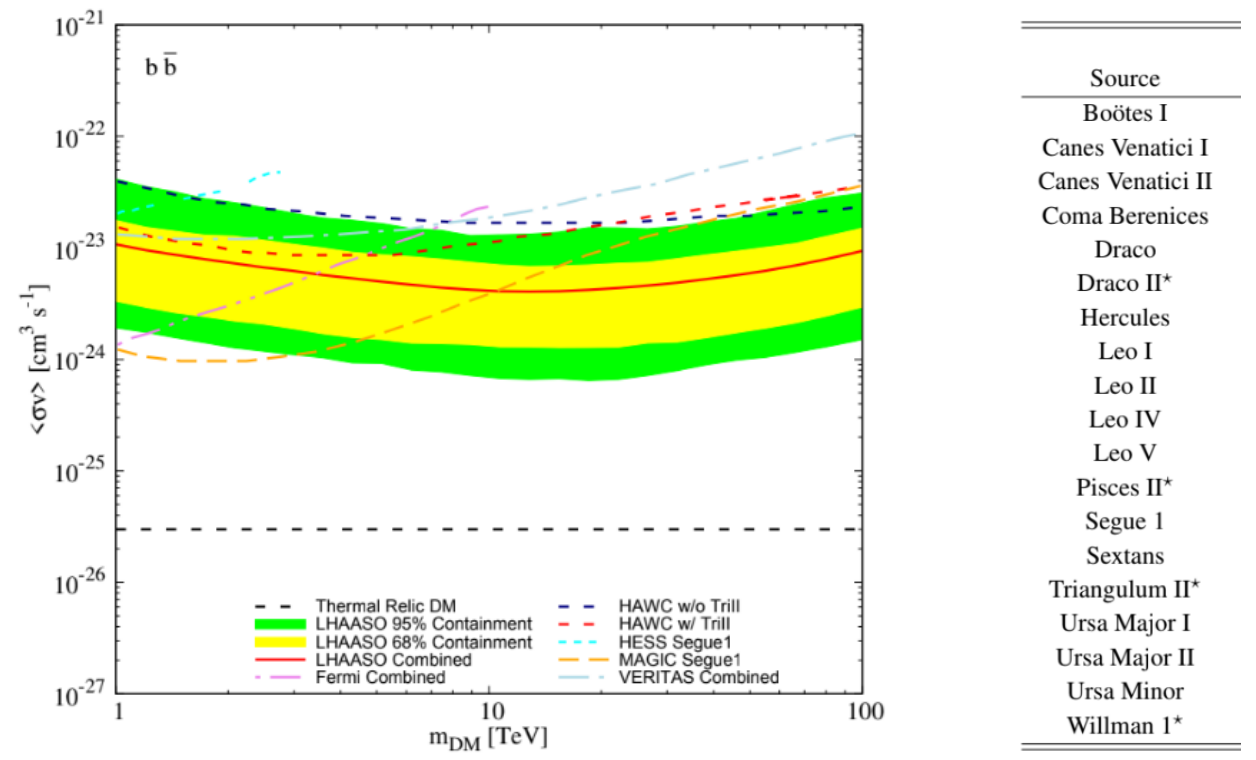

Figure 2: Left panel: Projected LHAASO median combined sensitivity (red solid line), as well as $68 \%$ and 95\% containment bands, for DM annihilation into $\bar{b} b$ final states, combined with various other constraints from dSphs. Right panel: The results are based on the list of dSphs shown in the right panel. From Ref. [8].

\subsection{WIMP searches at the Galactic center and the Milky Way halo}

One of the most compelling and theoretically best motivated DM candidate at $\mathrm{TeV}$ masses is Wino DM. Pure Wino DM is already now highly constrained by indirect searches, and might be hence at the verge of discovery with future searches. At the same time, it provides an important target for future colliders like, e.g., FCC-hh, making it excellent candidate for exploiting the complementarity between various WIMP DM search techniques.

The annihilation rate of Wino DM is Sommerfeld enhanced, due to the exchange of electroweak gauge bosons in the initial state. Higher-order corrections lead to pronounced endpoint features in the spectrum of Wino DM, including line components from $\chi_{0} \chi_{0} \rightarrow \gamma \gamma$ and $\chi_{0} \chi_{0} \rightarrow \gamma Z^{0}$, which further increase the sensitivity of indirect searches towards that model. Pure Wino DM constitutes all of DM today if its mass is around $m_{\mathrm{dm}} \sim 2.9 \mathrm{TeV}$.

In Ref. [10], forecasted limits for HESS observations of the Galactic center that are optimized for Wino searches were presented. In Fig. 3, the annihilation cross-section into monochromatic photons is shown compared to the expected upper limits from HESS. For a conventional cuspy Einasto profile, a $2.9 \mathrm{TeV}$ Wino is excluded by more than one order of magnitude in flux. However, for large DM cores, with radii of $\gtrsim 2 \mathrm{kpc}$, these limits become much weaker. In that case, Wino DM constitutes an excellent target for searches with the future CTA (Cherenkov Telescope Array). CTA is expected to improve existing constraints by an order of magnitude and more (in terms of statistics). The Wino annihilation signal is here a particularly good target since it features peculiar end-point features that will help to break through the difficulties of analysing diffuse astrophysical emission from the inner Galaxy. 

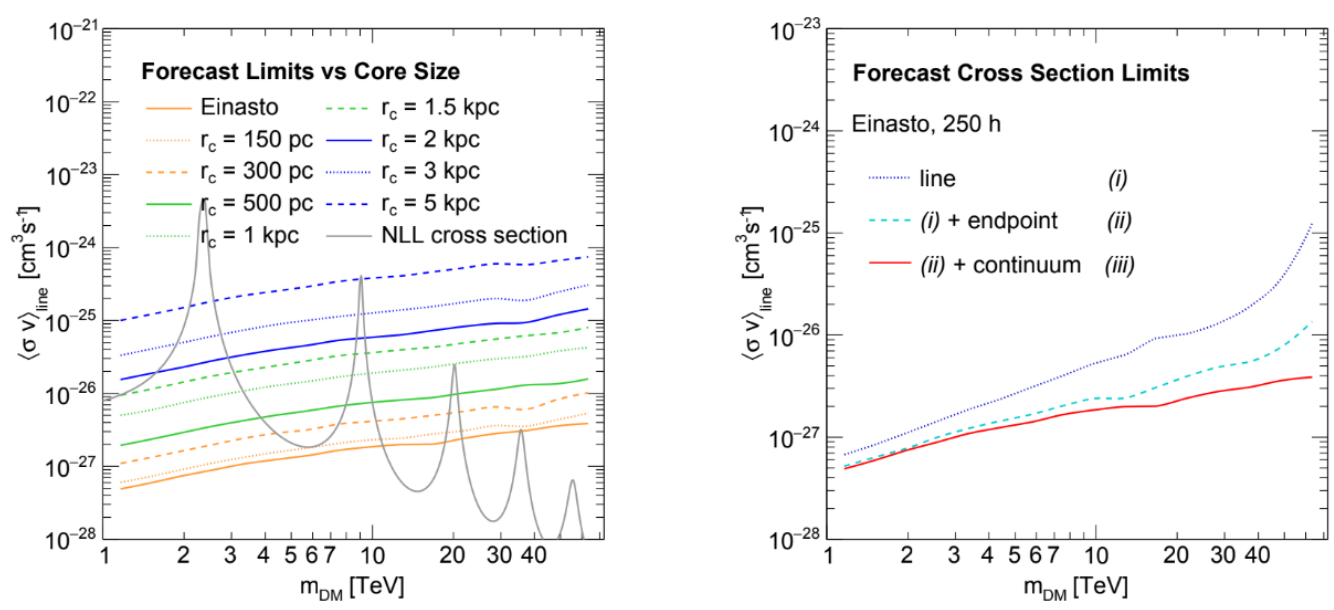

Figure 3: Left panel: HESS 95\% CL upper limits on the annihilation cross section into gamma-ray lines, $\langle\sigma v\rangle_{\text {line }}$, compared with theoretical predictions for Wino DM. Right panel: Expected strengthening of constraints when additional contributions to the endpoint feature as well as the continuum emission are included. From Ref. [10].

\subsection{Long-lived mediators}

Gamma-rays produced in the annihilation of DM particles that are trapped inside the Sun are immediately absorbed and cannot be observed. However, if DM instead annihilates into long-lived mediators $\phi$ (via $\chi \chi \rightarrow \phi \phi$ ), those can potentially leave the Sun before their subsequent decay into particles of the standard model (e.g., $\phi \rightarrow \gamma \gamma$ ). Searches for the associated gamma-ray emission can provide extremely stringent constraints on the annihilation rate within the Sun. In equilibrium, the annihilation rate is directly related to the capture rate, $\Gamma_{\text {ann }}=\frac{1}{2} \Gamma_{\text {cap. }}$. The capture rate is on the other hand a function of the spin-dependent, $\sigma_{\mathrm{SD}}$, and spin-independent, $\sigma_{\mathrm{SI}}$, WIMP-nucleon scattering cross-sections.

In Ref. [11], a search for this peculiar emission, using data from the Fermi-LAT, has been performed, using ON/OFF regions that co-move with the Sun. The process $\chi \chi \rightarrow \phi \phi$ with subsequent $\phi \rightarrow \gamma \gamma$ leads to box-like energy spectra, which extends from $E_{\gamma}=0$ to $E_{\gamma}=m_{\chi}$ in the limit $m_{\phi} \ll m_{\chi}$. Since such a spectrum is much harder than typical astrophysical backgrounds, the high-energy cutoff would be clearly visible as a spectral feature. Results of a spectral analysis and search are shown in Fig. 4, compared with previous findings from other work. For cascadeannihilating DM with $4 \gamma$ final states and mediators lifetimes that allow escape from the Sun core, the implied constraints on the spin-dependent WIMP-nucleon cross-section are many orders of magnitude stronger than what is possible with direct lab searches.

\subsection{Decaying dark matter}

If DM decays into particles of the Standard Model, cosmic- and gamma-ray observation can provide strong constrains on its lifetime, many orders of magnitude longer than the age of the Universe. Since the decay signal scales with the DM density $\propto \rho$ (rather than $\propto \rho^{2}$ in the case of annihilation), the best target for indirect searches are either massive objects like galaxy clusters, or 


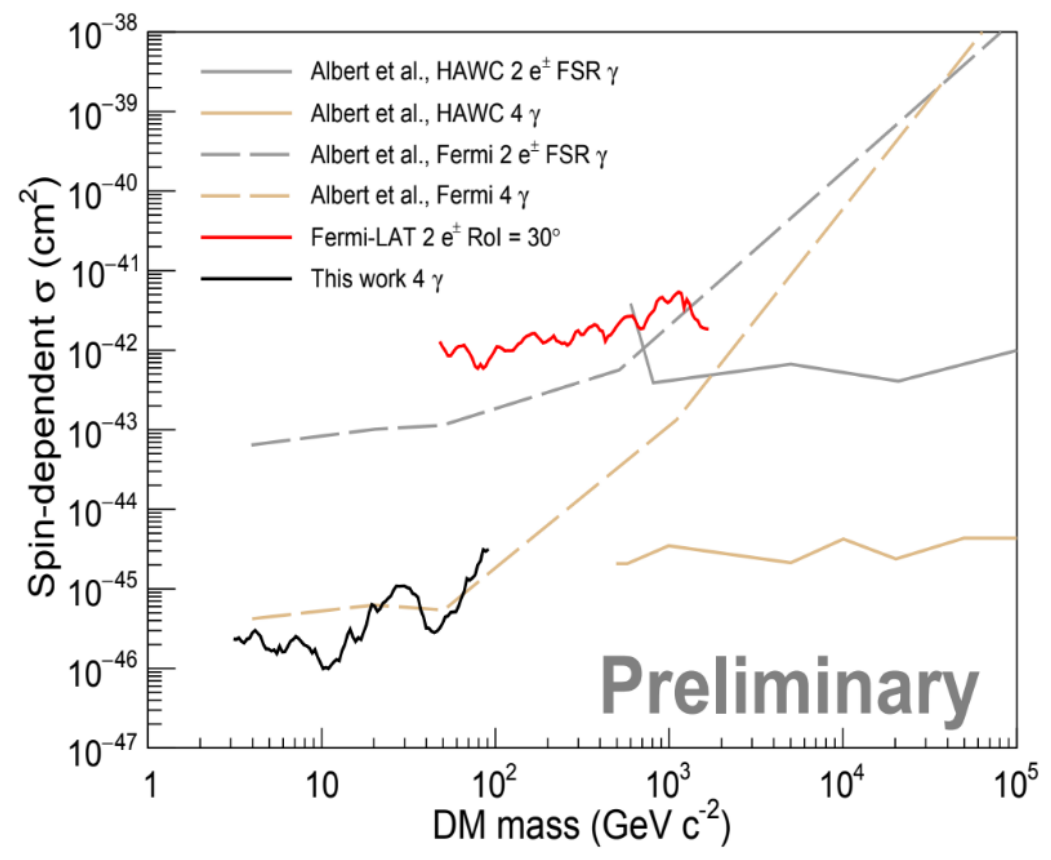

Figure 4: Limits on the spin-dependent WIMP-nucleon cross section for annihilation via long-lived mediators with a decay length $L=R_{\odot}$. Results based on Fermi-LAT data are shown as black line, together with constraints coming from a similar analysis using $e^{ \pm}$final states. Previous results from Fermi-LAT, based on less data, as well as HAWC, are shown as well. From Ref. [11].

directly the integrated emission from the Milky Way halo, which would typically be seen as mostly isotropic emission over the entire field-of-view of gamma-ray telescopes.

In Ref. [12], results from a recent dedicated Milky Way halo analysis using the HESS telescope have been presented. Since the analysis of diffuse emission is particularly challenging for IACTs like HESS, a novel observation strategy was developed to suppress background systematics. The idea is to pair up observations with exactly the same azimuthal and zeniths paths in the sky, but that correspond to different angular distance from the Galactic center. The former helps to minimize systematic differences in backgrounds, whereas the latter ensures a large differential in the expected DM signal flux (which can reach more than an order of magnitude). These differences can be used to cancel out background and other instrumental systematics. No significant emission correlated with the Galactic halo was found in 10 hours of HESS observations. The resulting lower limits on the DM lifetime are shown in Fig. 5. For DM masses in the range 30-200 TeV, DM lifetimes below $\tau_{\chi} \sim 10^{25} \mathrm{~s}$ are excluded. Similar constraints were obtained using observations of the Perseus cluster (using the MAGIC telescope).

Thanks to its large field-of-view $(\approx 2$ sr), HAWC is an excellent instrument to search for and constrain the lifetime of TeV DM particles decaying into particles of the Standard Model, since it allows large integration times for extended objects. In particular the Virgo galaxy cluster is an interesting target, covering approximately 3.6 degrees on the sky. The lower limits on the DM lifetime derived from HAWC observations of the Virgo cluster are shown in Fig. 6. They exclude $\mathrm{DM}$ lifetimes below $10^{27} \mathrm{~s}$ for a large range of $\mathrm{DM}$ masses from around a few $\mathrm{TeV}$ to $100 \mathrm{TeV}$ 


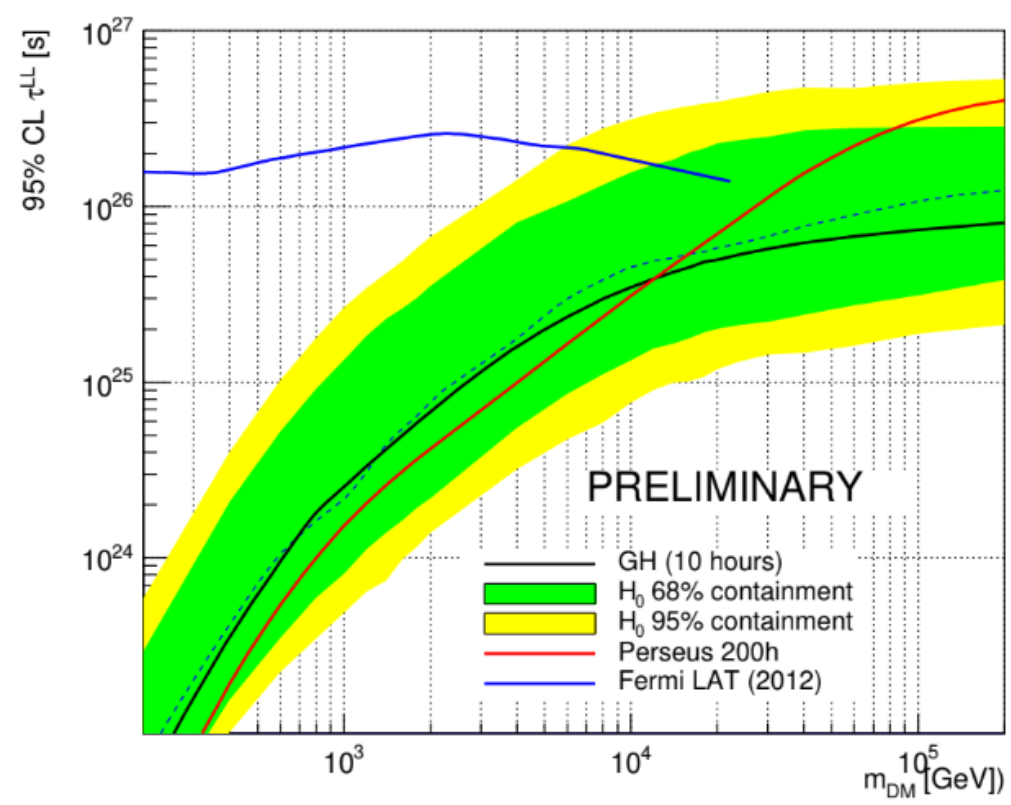

Figure 5: Lower limits on the lifetime for DM decaying $100 \%$ into $\bar{b} b$ final states, obtained by HESS observations of the Galactic Halo $(\mathrm{GH})$, using 10 hours of observation time and a new analysis strategy to suppress systematics. Constraints from Perseus cluster observations with MAGIC and Fermi-LAT constraints are shown as well. From Ref. [12].

(assuming annihilation into $\bar{b} b$ final states, but similar constraints are obtained for instance $\tau^{+} \tau^{-}$).

\subsection{Primoridal Black Holes}

Primordial black holes (PBHs) can be generated by large density fluctuations in the early Universe. Depending on the details of production, the can have in principle all possible masses between the Planck scale and super-massive black holes. BHs evaporate via Hawking radiation, with a temperature inversely proportional to the black hole mass. At temperatures above the QCD phase transition, $T \sim 250-300 \mathrm{MeV}$, they will start radiate quarks and gluons, which on astrophysical time-scales gives rise to a flux of photons, neutrinos, electrons, positrons, protons and anti-protons. These temperatures are expected to be reached around a Myr before the final evaporation. Although Hawking radiation is nearly negligible for stellar mass BHs, PBHs with an initial mass of $5 \times 10^{14} \mathrm{~g}$ are expected to evaporate in the current Universe, producing gamma-ray burst-like events observable at $\mathrm{GeV}-\mathrm{TeV}$ energies.

The HAWC observatory with its large instantaneous field-of-view is an excellent facility to search for the evaporation of PBHs. Ref. [14] presented results of a dedicated PBH evaporation search, building on top of a 959 day gamma-ray burst transient search with HAWC. The search was performed with sliding time windows of $0.2,1$ and $10 \mathrm{sec}$ duration. Taking into account trials factors correction due to the large field of view and time range, no signals were found. The resulting constraints on the burst rate density of PBHs are shown in Fig. 7. Constraints obtained from the various sliding time windows are similar, with the strongest constraints coming from the $0.2 \mathrm{~s}$ window. The emission model is based on the Standard Model particle content. HAWC improves 


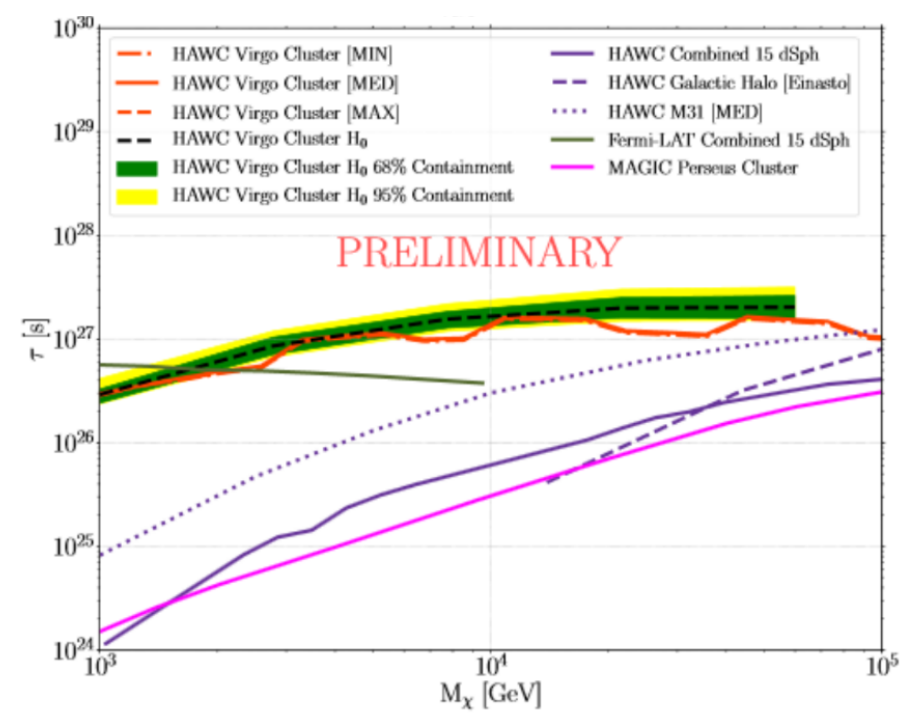

Figure 6: Lower limits on the DM lifetime, assuming decay into $\bar{b} b$ final states, based on HAWC observations of the Virgo cluster (red line), compared with constraints from other instruments. From Ref. [13].

previous constraints by Milagro, Veritas and HESS by about an order of magnitude, and constraints from Fermi-LAT (obtained by searches for moving gamma-ray sources) by a factor of a few.

In Ref. [15], it was argued that PBHs with masses between $10^{17}$ and $10^{21} \mathrm{~g}$ are good candidates for constituting all of DM. However, producing an sufficient amount of PBHs in the early Universe usually requires a significant amount of fine-tuning in the inflationary potential, since both very small density fluctuations at large scales (as seen in the cosmic microwave background, CMB) and density fluctuations of order one at small scales have to be simultaneously accommodated. Ref. [15] proposed alternative mechanisms for $\mathrm{PBH}$ generation related to the evolution and fragmentation of self-interacting scalar fields. Initial density perturbations are in this case completely decoupled from the PBH generation, making this scenario arguably more plausible.

In Fig. 8, constraints on fraction of DM that can be constituted of PBHs in various mass ranges are shown. To the left, constraints come from the non-observation of (continuous) Hawking radiation at gamma-ray energies. To the right, the strongest constraints come from micro-lensing searches for MACHOs (MAssive Compact Halo Objets) with the Hyper Suprime-Cam (HSC). PBHs that constitute a significant amount of DM in the allows mass range would lead to a large array of observable astrophysical signals, including GRB (gamma-ray urst) events from PBH-NS (neutron star) mergers, r-process production of heavy nuclei, and gravitational waves. The sensitivity of future searches is shown by the dashed lines in Fig. 8 .

\subsection{Axion-like particles and nuclearites}

The effects of a mixing between ALPs (axion-like particles) and photons on the gamma-ray emission of pulsars were studied in Ref. [16]. There, it was shown that for ALP-photon couplings close to helioscope constraints, the conversion in the extremely high magnetic fields close to neutron stars ( $\gamma \rightarrow \gamma^{*} a$, with $\gamma^{*}$ being part of the $B$-field) can be strong enough to alter the gamma-ray 


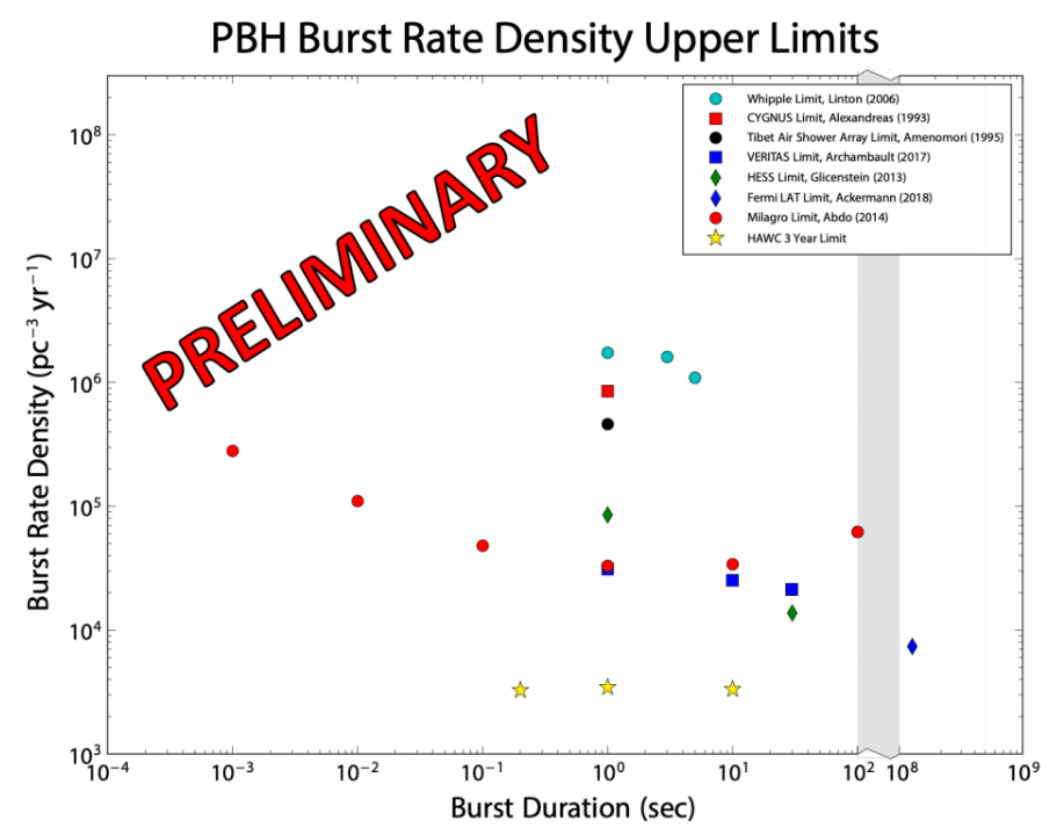

Figure 7: Constraints on PBH evaporation ratio from HAWC observations at $99 \%$ confidence level, compared with previous results. From Ref. [14].

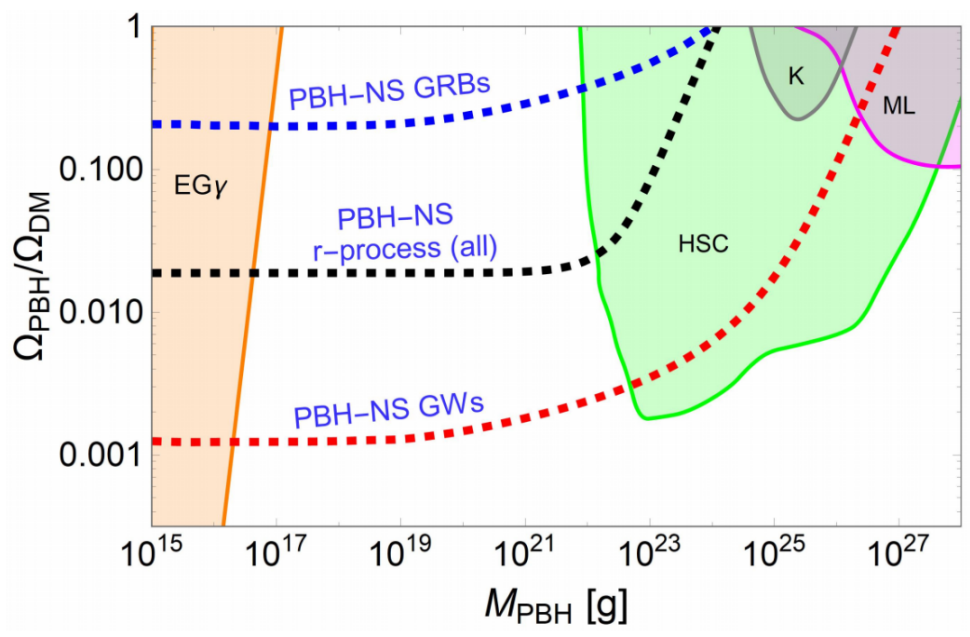

Figure 8: Signals from PBH-compact star interactions along with experimental constraints. From Ref. [15].

emission, although the effect might be hard to observe. A discussion about possible spectral modulations of the gamma-ray emission of Galactic pulsars due to photon-ALP mixing in the Galactic magnetic field was presented in Ref. [17].

Nuclearites are hypothetical massive nuggets of strange quark matter (SQM). The hypothetical SQM is composed of equal numbers of up, down and strange quarks, electrically neutral, and presumably stable for any Baryon number / mass. Although the existence of SQM remains speculative, it is interesting to consider the phenomenological consequences if is constitutes a significant fraction of DM in the Universe. When nuclearites traverse matter, a part of the energy 
loss due to elastic scattering with atoms is transformed into light. In the atmosphere, this would cause meteorite-like events, moving light-spots, which however do not 'burn out'r Typically, they would have velocities significantly larger than meteorites bound to Sun's gravity. Results from preformance tests of various high-sensitivity CMOS cameras for DM nuclearite and interstellar meteorite searches were presented in Ref. [18]. Observations using 4 CMOS cameras located at the Telescope Array side in Utah, USA, are planned for in the next year.

Ref. [19] showed first preliminary results from nuclearite searches with the TUS (Tracking Ultraviolet Setup) air fluorescence detector aboard the Lomonosov satellite (launched in 2016). Monte Carlo results for nuclearites with masses between $100 \mathrm{~g}$ and $100 \mathrm{~kg}$ were presented. Current sensitivities are two low to be sensitive to expected DM rates. However, the results provide preparatory work for similar analysis with, e.g., the proposed JEM-EUSO fluorescence detector, currently planned for after 2020. Finally, constraints on the flux of 'heavy stable macro particles" (including nuclearites and meteorites), based on the 'Pi of the Sky' project, were presented in Ref. [20].

\section{Dark matter searches with neutrinos}

Self-annihilation of WIMP DM in general produces numerous of high-energy neutrinos. In the case of hadronic final states, they mostly stem from the decay of charged pions, typically with a total deposited energy of the order of photons (mostly coming from neutral pion decay). Although high-energy neutrinos are significantly more difficult to detect than gamma-rays and charged cosmic-rays, neutrinos are an important channel for DM searches for at least two reasons: First, given our ignorance about the DM annihilation channels, neutrino might play a significant role and other radiation components might be suppressed. Second, given their feeble interactions, they can escape stellar and planetary objects, which makes them an important messenger for the annihilation of DM that is gravitationally trapped within these objects.

\subsection{Galactic center searches for WIMPs and decaying DM}

Constraints on the annihilation cross-section into neutrino final states were presented before by the ANTARES and IceCube instruments. ANTARES is an underwater Cherenkov detector in the Mediterranean sea, situated near Toulon. It consists of 12 detection lines, each equipped with multiple photo-multiplier cameras enclosed in optical modules. IceCube is a cubic-kilometer neutrino detector located at the geographic South pole, at a depth of around $2 \mathrm{~km}$. It consists of 5,160 digital optical modules (DOMs), which are distributed over 86 string. The central eight strings are deployed compactly, in the 'DeepCore' subdetector.The planned upgrade in 2022/2023 will consists of 7 new strings with about 700 additional optical detectors, densely (with a $3 \mathrm{~m}$ separation) located inside DeepCore. This will extend the accessible neutrino energy range down to $1 \mathrm{GeV}$.

In Ref. [21] the first results from a joined likelihood analysis of Galactic center data from both telescopes was presented. This was based on 9 years of ANTARES and 3 years of IceCube data. Data samples were combined at the likelihood level, and no significant excess above expected backgrounds was found. Such a combination is interesting for a number of reasons. First, it allows to take into account systematics related to the signal strength in a statistically sound way. Second, 


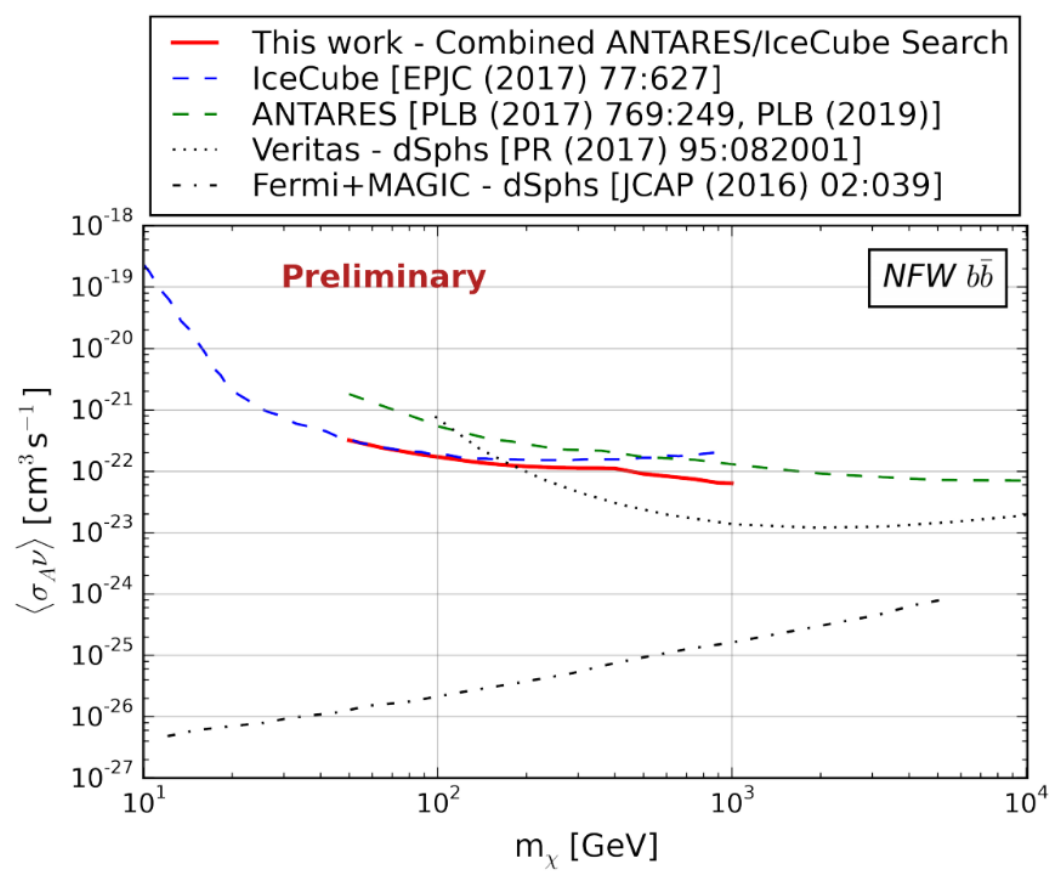

Figure 9: Combined constrains on dark matter annihilation into neutrinos from Galactic center observations with IceCube and ANTARES. From Ref. [21].

since IceCube is situated in the South, and ANTARES in the norther hemisphere, the view on the Galactic center is very different: ANTARES sees the Galactic center through the Earth, and is hence not affected by backgrounds from atmospheric neutrinos.

The resulting constraints on the annihilation cross-section for $\chi \chi \rightarrow v v$ shown in Fig. 9. At high energies, the constraints are dominated by ANTARES, whereas at low energies (below $m_{\chi} \sim$ $500 \mathrm{GeV}$ ), the limits are dominated by IceCube observations.

In the near future, KM3NeT is expected to significantly increase the reach towards neutrinos from WIMP annihilation. KM3NeT, currently under construction in the Mediterranean see, will consist of two components: ORCA (Oscillation Research with Comics in the Abyss), which is optimized for oscillation research with atmospheric neutrinos. ARCA (Astroparticle Research with Comics in the Abyss) is optimized for high energies. Both detectors are currently deployed, and will contain a total area of about one cubic-km. The sensitivity reach with KM3NeT is shown in Fig. 10, where the expected sensitivity of the ARCA detector of KM3NeT (assuming one year of observation) is compared with current constraints from ANTARES (11 year of observation time). The expected improvement is more than one order of magnitude, and for neutrino final states the constraints almost approach the thermal cross-section for DM masses around $1 \mathrm{TeV}$.

Plans for the search for DM with the IceCube Upgrade were discussed in Ref. [23]. At 10 $\mathrm{GeV}$ energies, the effective area will increase by more than an order of magnitude, and detections extend down to around $1 \mathrm{GeV}$. This will allow to improve current constraints on DM annihilation into neutrinos, e.g., $\chi \chi \rightarrow v_{\mu} \bar{v}_{\mu}$, by more than an order of magnitude for DM masses $m_{\chi} \lesssim 10 \mathrm{GeV}$. 


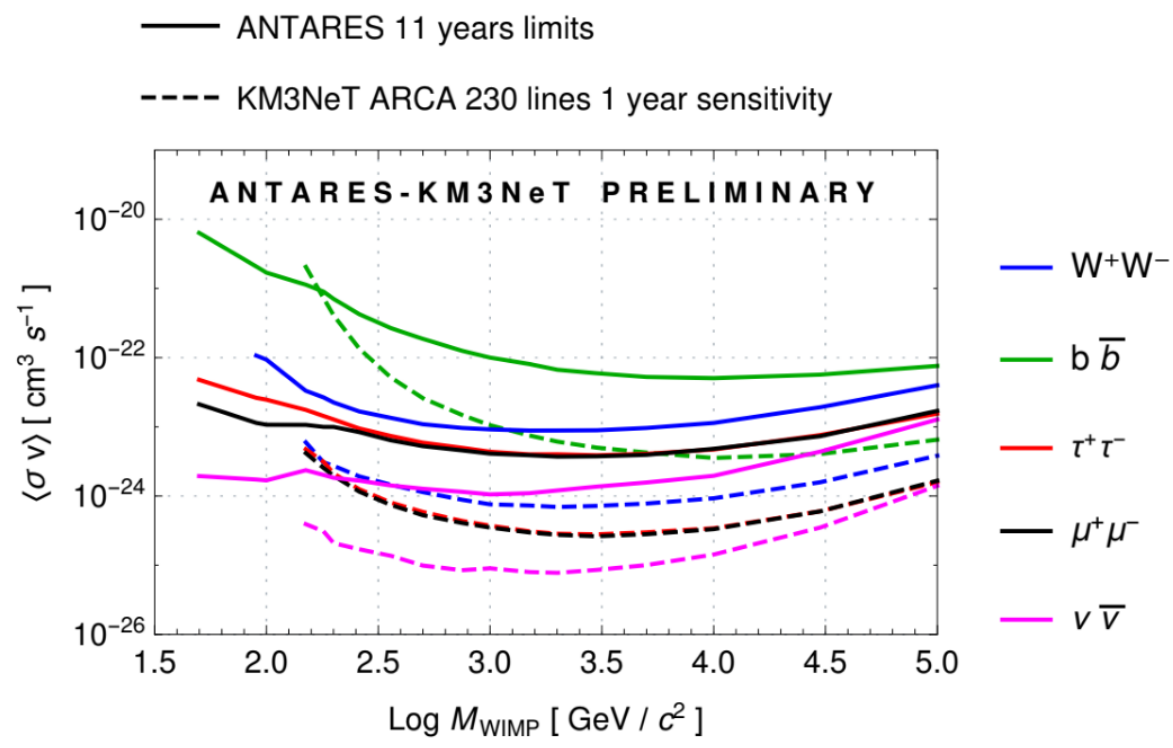

Figure 10: Expected sensitivity of the ARCA detector of KM3NeT for WIMP DM annihilation into various final states. Already after one year of observation KM3NeT will improve upon 11 years of ANTARES observations by one order of magnitude. For $\chi \chi \rightarrow \bar{v} v, \mathrm{KM} 3 \mathrm{NeT}$ is expected to close to the thermal crosssection. From Ref. [22].

\subsection{WIMP searches with Sun and Earth targets}

The Sun and Earth are prime targets for DM searches with neutrino cosmic-rays. The annihilation rate of WIMPs gravitationally trapped inside these objects can be very high, depending on the WIMP capture rate (which is related to the WIMP-nucleon cross-section). The associated annihilation signal can however only be seen in neutrinos, since other Standard Model particles would be absorbed by the Sun's plasma. A few updates on these kind of searches were presented.

Sensitivity studies for Solar DM signals with the KM3NeT-ORCA detector were presented in Ref. [24]. ORCA observations will be able (assuming 5 years observation time) to improve upon current IceCube constraints on the WIMP-nucleon cross section by factors of a few to an order of magnitude, in the WIMP mass range $20-100 \mathrm{GeV}$.

Planned improvements on the IceCube Solar WIMP search were presented in Ref. [25], including systematic comparisons between event generators and improved event selections. Methods for searches for DM annihilation signals from the center of the Earth with 8 years of IceCube data were presented in [26].

\subsection{Secluded DM, PeV neutrinos, an nuclearite searches}

Sensitivity estimates for IceCube searches for secluded DM with long-lived mediators were shown in Ref. [27]. Here, the Sun was used as target. For conventional WIMP annihilation, the neutrino signal from the Sun is strongly attenuated due to interactions with the solar plasma, especially at high energies. In secluded DM scenarios, the mediator could however leave the Sun before decaying into neutrinos, producing a much harder signal spectrum. It was shown that with 6 years of data IceCube can be sensitive to spin-dependent WIMP-nucleon cross-sections down 
to $3 \times 10^{-34} \mathrm{~cm}^{2}$ for TeV DM masses. On the other hand, Ref. [28] presented the prospects to use ANTARES for searches for the specific neutrino energy spectra predicted by secluded DM, for DM masses up to $200 \mathrm{TeV}$, and using the Galactic center as target. Since the mediator lifetime has to decay within 0.1s or so (because of Big Bang Nucleosynthesis constraints) the morphology of secluded DM signals would be similar to regular WIMP annihilation.

In Ref. [29], indications for a multi-component TeV-PeV neutrino spectrum based on a comparison between different IceCube measurements and ANTARES results are presented. A possible explanation for an excess in the range 40-200 $\mathrm{TeV}$ are contributions from DM annihilation or decay. Future observations of the angular distribution of the emission with KM3NeT and significantly improved statistics will allow to probe this scenario with high statistical significance, provided the excess can be completely attributed to decaying DM.

\section{Dark matter searches with positron cosmic rays}

Dark matter annihilation/decay, in particular into leptonic final state, contributes to the cosmicray electron and positron fluxes. The clear observation of the rise in the positron-to-electron fraction above $10 \mathrm{GeV}$ triggered significant interest since its cause could be DM (although the high required annihilation/decay rate would be usually in conflict with the non-observation of signals in gamma-ray observations). However, $e^{ \pm}$pairs produced in pulsar magnetospheres are a plausible astrophysical explanation. In Ref. [30], it was demonstrated that the observed positron excess can be fully explained by contributions from the Geminga pulsar if a two-zone diffusion model is assumed.

\subsection{Below $1 \mathrm{GeV}$}

The major obstacle for indirect DM searches with charged cosmic rays with energies below $\sim 5 \mathrm{GeV}$ is the strong impact of the solar wind which acts as a cutoff of the energy spectra. Most interestingly, Voyager-1, which was launched in 1977, has crossed the heliopause, which defines the boundary between solar wind and interstellar medium, in summer 2012. Since then, it provides information about the interstellar cosmic-ray spectra, which can be used to constrain DM models. The resulting constraints are relevant DM masses below $\sim 1 \mathrm{GeV}$, and in particular for scenarios with $p$-wave annihilation that are less constrained by observations of the CMB.

In Ref. [31], the Voyager-1 measurements of the $e^{ \pm}$spectrum at energies from around 10 $\mathrm{MeV}$ to $50 \mathrm{MeV}$ (shown in Fig. 11), were used to constrain DM annihilation as well as PBH evaporation. Limits approach annihilation cross sections of $\langle\sigma v\rangle \sim 10^{-28} \mathrm{~cm}^{3} \mathrm{~s}^{-1}$ for light DM with masses $m_{\chi} \sim 10 \mathrm{MeV}$, which is competitive with gamma-ray probes. For $p$-wave annihilation the resulting constraints are significantly stronger than other astrophysical probes including the CMB. The Hawking radiation of PBHs with masses below $10^{16} \mathrm{~g}$ would also significantly affect the measured electron spectrum, and their contribution to the overall DM density can be constrained to less than $0.1 \%$ at these masses. Although the constraints are not affected by the solar wind, uncertainties related to cosmic ray propagation in the interstellar medium remain within an order of magnitude. 

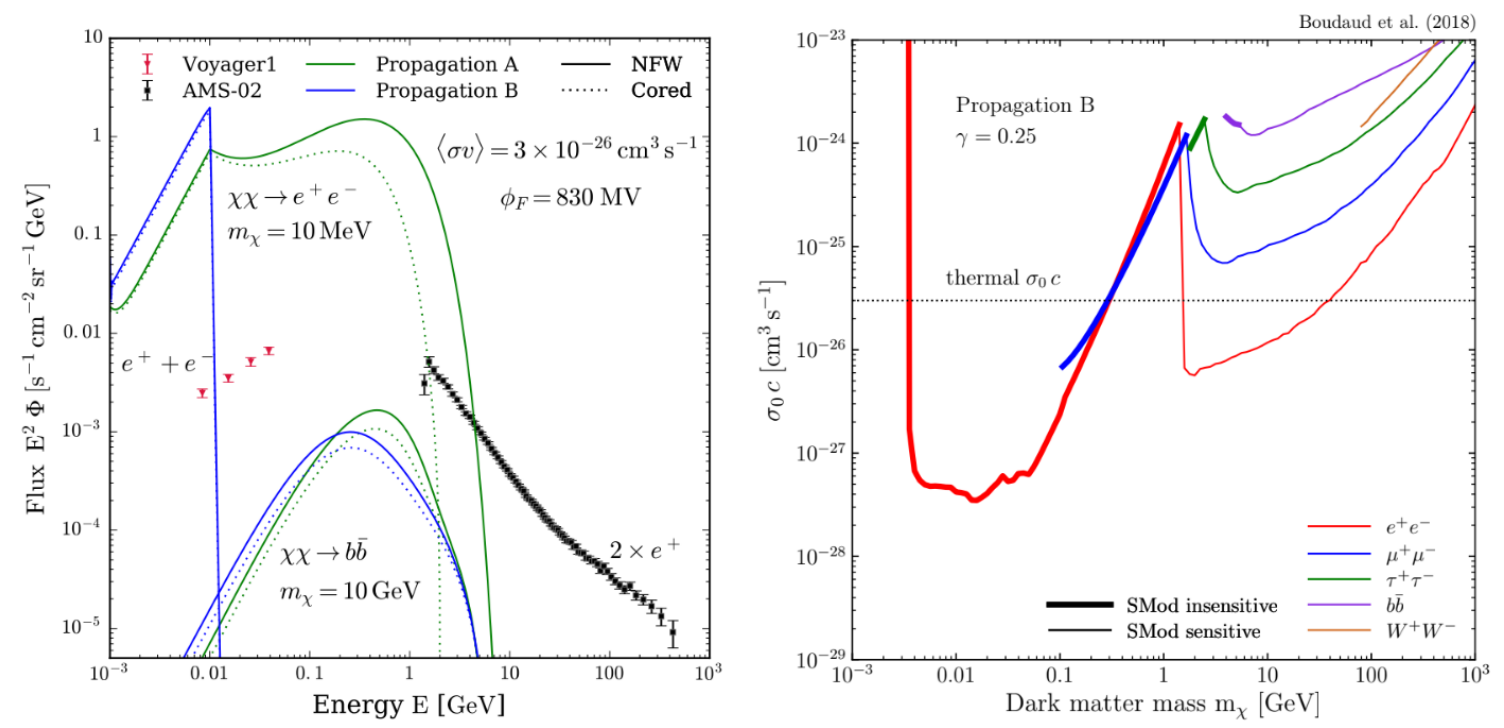

Figure 11: Left panel: The $e^{ \pm}$flux of cosmic rays, measured by Voyager-1 and AMS-02, compared with contributions from DM annihilation for different CR propagation scenarios and DM profiles. Right panel: Upper limits on the $p$-wave annihilation cross-section derived from Voyager-1 data, based on a CR propagation scenario with strong re-acceleration, compared with the thermal value, for different final states. From Ref. [31].

\subsection{Above $1 \mathrm{GeV}$}

DM annihilation into leptonic final states can give rise to peculiar spectral features in the $e^{ \pm}$ cosmic-ray spectrum, at energies around the DM mass. The non-observation of such features in CR measurements can be used to put strong constraints on the DM annihilation into leptonic final states. In Ref. [32], an updated search for such spectral features was performed using more than 10 years of Fermi-LAT data. Typical spectra are bumps, peaks or box-like spectra on top of otherwise smooth cosmic rays. In order to isolate potential features, a sliding energy window technique was used. The energy spectrum of the measured CRs allow to search for DM with masses from $70 \mathrm{GeV}$ to $1.7 \mathrm{TeV}$. No significant excess was found, and instead constraints derived, as shown in Fig. 12. The constraints intersect the thermal annihilation rate at masses just above $m_{\chi} \approx 100 \mathrm{GeV}$, and hence exclude thermal $s$-wave annihilation via $\chi \chi \rightarrow e^{+} e^{-}$below that mass.

For conventional WIMP annihilation or decay, the signal flux is expected to be largely isotropic. However, in scenarios with long-lived mediators (see Sec. 2.3), it is possible that the signal is dominated by $e^{ \pm}$pairs produced just outside the Sun by mediator decay. Dedicated searches for these kind of signals have been performed as well, concentrating on a $30^{\circ}$ region around the Sun. No signal was found, and the resulting constraints are similar to Fig. 4 [32].

A similar analysis was performed in Ref. [33], using data from the CALET experiment, that covers roughly $10 \mathrm{GeV}$ to $4 \mathrm{TeV}$. The CALET (CALorimetric Electron Telescope) detector is attached to the International Space Station since 2015, and measures $e^{ \pm}$up to $\mathrm{TeV}$ energies, with good spectral/spatial resolution and high proton rejection rates. Interestingly, the measured $e^{ \pm}$data shows some spectral variations around $350 \mathrm{GeV}$ that can be interpreted in terms of annihilation of DM with a similar DM mass. However, contributions from multiple pulsars with different cooling 


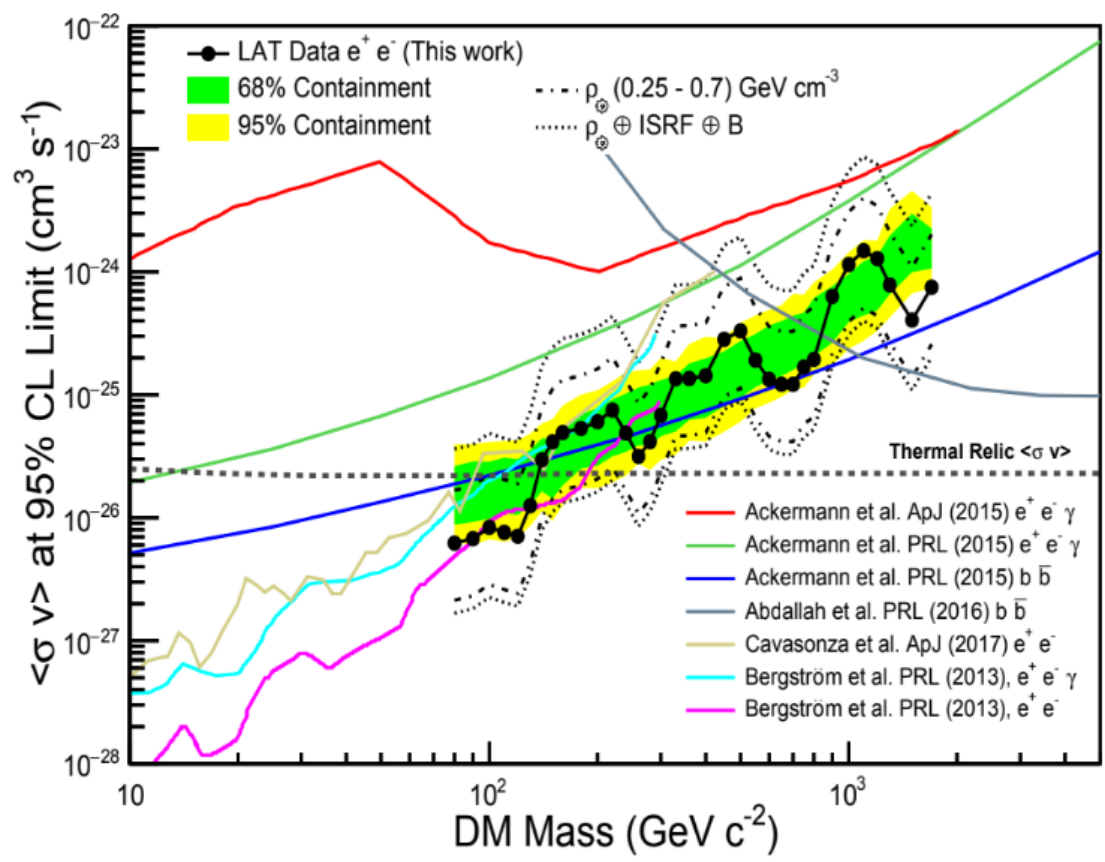

Figure 12: Updated upper limits on the annihilation cross-section for $\chi \chi \rightarrow e^{+} e^{-}$, as obtained from a spectral analysis of the electron plus positron cosmic-ray spectrum measured by Fermi-LAT after 10 years. Variations with different magnetic fields and local dark matter densities are shown as well, together with results from previous similar analysis. From Ref. [32], where further details can be found.

cutoff in the spectrum, and the inclusion of systematic uncertainties, render the observed spectral variation insignificant. The corresponding constraints are similar (though somewhat weaker) than the ones obtained from Fermi-LAT data.

\section{Dark matter searches with anti-nuclei cosmic rays}

In Ref. [34], the status of DM searches with cosmic-ray antinuclei was summarized. Dark matter annihilation into hadronic final states would produce a significant amount of anti-matter (including positrons, anti-protons and anti-deuterium), and contribute potentially visibly to the measured cosmic-ray fluxes. Besides searches for annihilation or decay contributions to the cosmic-ray flux, the scattering of DM particles on primary cosmic rays could alter the spectrum provided large enough energy transfer. This effect was discussed in Ref. [35].

\subsection{Anti-protons}

Anti-proton cosmic-rays are an excellent target for DM searches for a number of reasons. First, the astrophysical backgrounds from secondary (and tertiary) production of anti-protons in cosmic-ray collisions with the interstellar medium is relatively low. Second, this background can be reasonably well predicted, usually within a factor of less than two, from the measured primary cosmic ray spectra as well as other secondary-to-primary ratios (in particular the Boron-to-Carbon ratio). When calculating constraints on DM, the arguably largest uncertainty comes from the exten- 


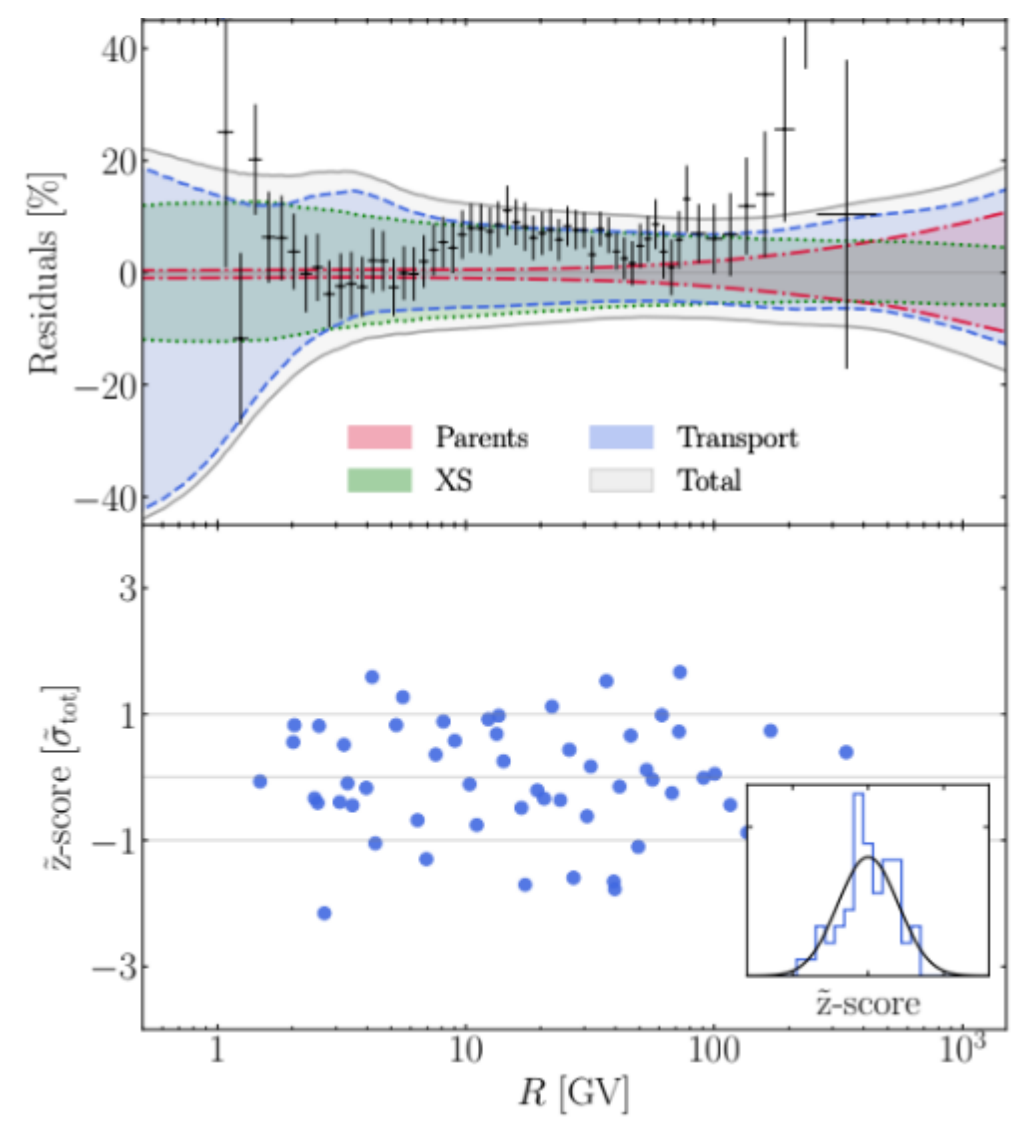

Figure 13: Top panel: Difference between measured anti-proton flux (AMS-02) and the predicted astrophysical background, together with the source of various systematics uncertainties in the background determination (primary cosmic rays, production cross-section, cosmic-ray transport). Bottom panel: Statistical significance of deviations from the predicted background. No significant excess is observed beyond the assumed systematics. From Ref. [36].

sion of the diffusion halo of the Galaxy, which also defines the 'collection area', where anti-protons generated in DM annihilation cannot freely escape the irregular Galactic magnetic field.

Since a couple of years, there has been a debate about a possible excess of anti-protons at rigidities around $10 \mathrm{GV}$. The excess itself is of the order of a few percent of the background, which is at the level of systematic uncertainties of AMS-02. This implies that understanding these systematics, as well as the systematic uncertainties in the secondary cosmic-ray production, is of high relevance to claim an excess or refute it. Subsequent work, indicates that the statistical significance of the excess depends on what assumptions are made about cosmic-ray propagation, the anti-proton production cross-section as well as the correlated systematics of the anti-proton measurements themselves.

In any case, an interesting aspect of this anti-proton excess is that it would be compatible with DM annihilation into $\bar{b} b$ final states and a DM mass around $m_{\chi} \sim 50-80 \mathrm{GeV}$ and a closeto-thermal cross-section. This is compatible with the DM interpretation of the Fermi-LAT GeV excess seen in the Galactic center. It is rather intriguing that anti-protons could have largely ruled 


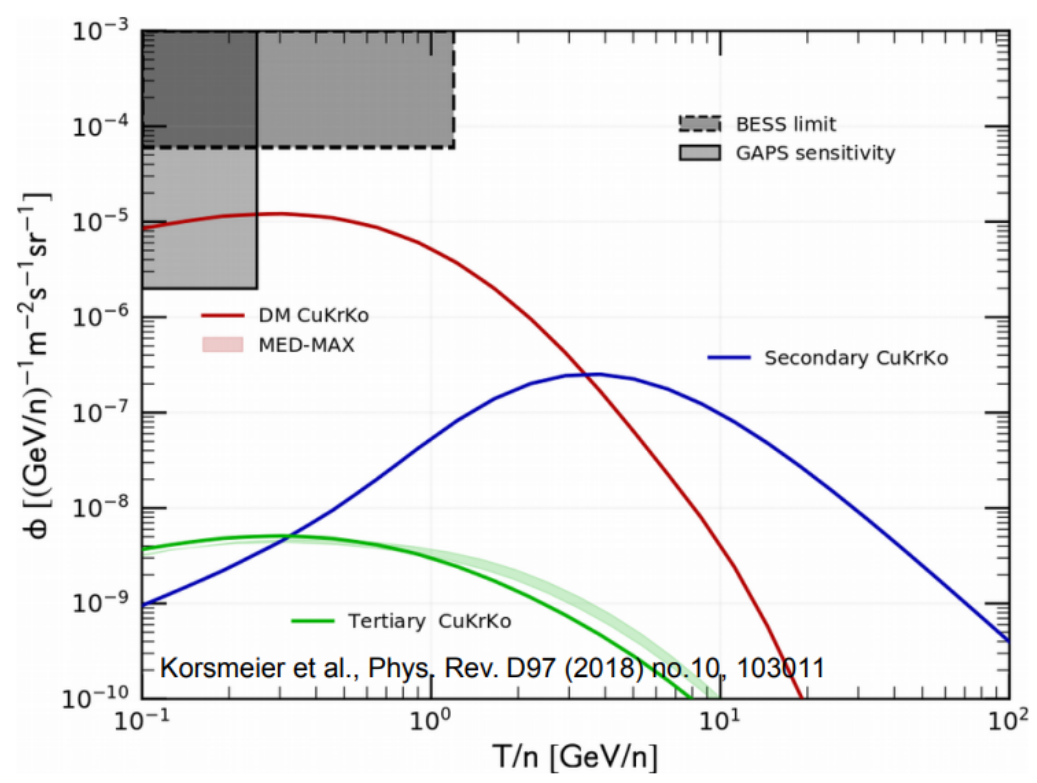

Figure 14: GAPS sensitivity of three 35-days flights for anti-deuteron flux, compared with new limits from BESS-Polar-II and the expected flux for the DM interpretation of the anti-proton excess. See Ref. [34].

out the Fermi GeV excess as a DM signal, but the excess in AMS-02 data weakens the constraints to such a degree that the exclusion is not possible.

In Ref. [36], results from a new analysis of the AMS-02 anti-proton measurements were presented. The authors carefully account for a large range of correlated systematics and propagate them through to the predicted astrophysical anti-proton background. The results are shown in Fig. 13. A caveat of the analysis is that the correlation structure of many of the uncertainties, in particular the ones related to the measurement itself, had to be estimated since they are not public information. In any case, the predicted (not fitted) anti-proton background is in good agreement with the measurements at the level of 5-10\%, which is also the level of uncertainties related to primary cosmic-ray spectra, the production cross-section and cosmic-ray transport uncertainties. Within this framework, no significant excess could be identified.

\subsection{Anti-deuteron}

As we saw above, although secondary backgrounds for anti-protons can be reasonably well predicted, the small statistical errors that AMS-02 measurements achieved make it necessary to accurately quantify all relevant background and measurement systematics, which can be a tedious and error-prone task. This caveat does not apply to anti-deutrons as target for DM searches, since the expected backgrounds from secondary and tertiary production via cosmic-ray interactions with the interstellar medium are expected to only contribute negligibly to upcoming measurements. The detection of a signal would constitute a smoking-gun signature for DM annihilation or decay.

In Fig. 14, the expected sensitivity of the planned GAPS balloon mission is shown, compared to previous constraints from the BESS balloon. It can be seen that DM annihilation as an explanation for the anti-proton excess around $10 \mathrm{GV}$ predicts a significant and detectable contribution to the anti-deuteron flux. It should be detectable by GAPS after a few observational rounds. The 
first flight for GAPS is scheduled for 2021. A study of the event-reconstruction performance of the GAPS experiment was shown in Ref. [37]

\section{Lab searches for DM}

At ICRC 2019, also a few updates and new results from the direct search for DM particles with lab experiments were presented, including DM Ice-17, COSINE-100, DAMIC and FUNK, which we discuss below. Furthermore, the BSUIN project (Baltic Sea Underground Innovation Network), which investigates new opportunities for underground laboratories around the Baltic sea, was presented in Ref. [38].

\subsection{DAMIC-M, DM Ice-17 and COSINE-100}

Results from the DM-Ice17 and COSINE-100 experiments were presented in Ref. [39]. The DM-Ice17 experiment is located at the South Pole within the IceCube detector, and consists of two $8.5 \mathrm{~kg} \mathrm{NaI}(\mathrm{Tl})$ crystals. Goal of the experiment is to probe the detection of an annual modulation signal in the DAMA detector with the same target material but in an environment with completely different backgrounds and different DM signal phase. This prototype study demonstrated that such an experiment is feasible, but the acquired data over $31 / 2$ years is statistically not sensitive enough to probe the DAMA signal. On the other hand, the COSINE-100 detector, with $8 \mathrm{NaI}(\mathrm{Tl})$ crystals (106 kg in total), located at the Yangyang underground laboratory in South Korea, has collected during the last three years enough data to exclude the simplest WIMP explanations of the DAMA signal with the same target material. The annual modulation analysis is still ongoing and will require a few years additional data before delivering conclusive results. In the case a signal is observed, plans for a much larger DM-Ice250 experiment are discussed, potentially in context of the IceCube upgrade 2022/2023.

The DAMIC (Dark Matter in CCDs) detector at SNOLAB in Ontario Canada is a cryogenic detector that uses high resistivity CCDs to search for light DM particles below masses of $10 \mathrm{GeV}$. In Ref. [40], a detailed discuss about background modeling and preliminary results for constraints on the WIMP-nucleon coupling were presented. Already in its current form, DAMIC at SNOLAB will be able to deliver constraints around a few GeV WIMP mass that are similar to results from other detectors. Numerous of upgrades are planned, including the use of skipper CCDs to significantly reduce the CCD readout noise, and the construction of the larger DAMIC-M (at Modane). The latter is expected to improve constraints on the WIMP-nucleon cross-section for DM masses below $5 \mathrm{GeV}$ by $1-2$ orders of magnitude with respect to existing experiments.

\subsection{Dark photon searches with FUNK}

Massive hidden photons are a valid candidate for dark matter with many interesting phenomenological properties and good theoretical motivation. They would correspond to the gauge bosons of an extra $U(1)$ local symmetry beyond the gauge symmetry structure of the standard model. At low energies, hidden photons would couple exclusively to the standard model photon via a (weak) mixing in the kinetic terms. This induces a weak coupling between electrically charged particles and the hidden photon. In this scenario, hidden photons can be non-thermally produced in the early Universe and survive as cold dark matter until the present Universe. The 


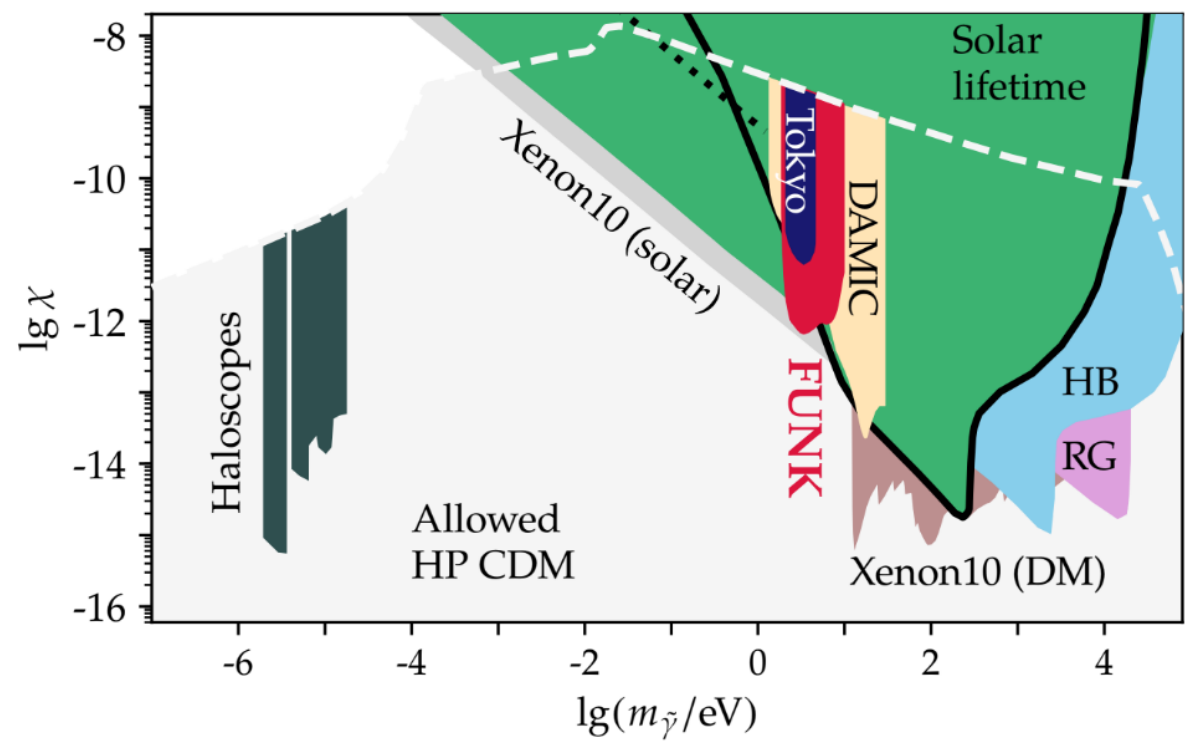

Figure 15: Exclusion limits (at 95\% CL) obtained by the FUNK experiment on the kinetic mixing parameter $\chi$ between hidden and visible photons, compared to other experimental constraints. From Ref. [41].

only parameters of this model are the hidden photons mass, $m_{\tilde{\gamma}}$, and the kinetic mixing parameter, $\chi$. A large region of the parameter space would be compatible with hidden photon cold dark matter, which is experimentally probed by haloscopes, direct detection experiments and various astrophysical observations.

In Ref. [41], results from a dedicated search for hidden photons with spherical mirrors were presented, using the FUNK experiment (Finding $U(1)$ s of Novel Kind). The kinetic mixing between hidden and visible photon results in a certain probability to emit a measurable photon when a hidden photon hits the mirror surface. Due to the differences in mass, the visible photons would be reflected back almost perpendicular to the mirror surface, which suggests the use of spherical mirror for optimal signal collection. The signals are measured with dedicated photo multiplier tubes with an extended sensitivity range from 160 to $630 \mathrm{~nm}$. The measurement was performed using various settings with in/out of-focus detectors and closed/open shutters. No signal was found, and constraints on the kinetic mixing parameter were obtained for hidden photon masses between $1.94 \mathrm{eV}$ and $8.40 \mathrm{eV}$, that are comparable with other constraints from astrophysical observations and the Xenon-10 detector, see Fig. 15.

\section{Conclusions}

About 70\% of the dark matter contributions to the ICRC 2019 presented updates and new ideas for WIMP searches. A notable development for indirect DM searches is a trend towards the combination of different experimental data at the collaboration level. Indeed, combined likelihood approaches with different targets and experiments were presented in Ref. [2] for gamma-ray observations (with Fermi, HESS, MAGIC, Veritas and HAWC, see Fig. 1) and in Ref. [21] for observations of cosmic-ray neutrinos (with ANTARES and IceCube, see Fig. 9). These combinations allow to both increase the sensitivity in regions of the parameter space where the sensitivity 
of multiple experiments is comparable, and to eliminate systematic differences in the results published by different groups. In particular, Ref. [2] excluded s-wave annihilation of WIMP DM into $\bar{b} b$ final states for DM masses below $m_{\chi} \approx 100 \mathrm{GeV}$. Among the various WIMP candidates, Wino dark matter played a special role, due to its high expected signal flux and relevance for current (HESS) and future (CTA) indirect dark matter searches [10]. Furthermore, the planned LHAASO observatory will be able to improve on constraints on heavy DM, $m_{\chi} \gtrsim 10 \mathrm{TeV}$, based on $\mathrm{dSph}$ observations, by a factor of up to five over current IACT results.

New limits on decaying DM were obtained by dedicated Galactic Halo observations with HESS, based on a novel observational pattern to suppress background systematics. The lower limits reach up to $10^{26} \mathrm{~s}$ for DM masses above $10 \mathrm{TeV}$, see Fig. 5. Those limits are surpassed, however, by HAWC observations of the Virgo cluster, by a factor of around ten [12], see Fig. 6, which can be attributed to the much longer effective integration time due to HAWC's large field-ofview. Cascade annihilating DM via long-lived mediators was discussed in multiple contributions. For instance, in Ref. [11], strong limits on the spin-dependent WIMP-nucleon scattering crosssection were derived, based on searches for box-like spectra in Fermi-LAT data towards the Sun (under the assumption that the mediator is long-lived enough to escape the Sun, see Fig. 4).

A possible excess at around $10 \mathrm{GV}$ in the anti-proton measurements of AMS-02 was reexamined in Ref. [36], taking into account various background and instrumental systematics; as a result, the existence of a statistically significant excess could not be confirmed, see Fig. 13. However, the DM interpretation of the $10 \mathrm{GV}$ excess will be soon tested by anti-deuterium measurements with the GAPS balloon experiment [34], which is scheduled for first flight in 2021, see Fig. 14.

The excess of positron cosmic rays above $10 \mathrm{GeV}$ was not directly debated as DM candidate at the conference. However, various spectral analyses of the $e^{+}$and $e^{ \pm}$cosmic-rays above $100 \mathrm{GeV}$, as measured by Fermi-LAT and CALET, were presented, and exclude $s$-wave annihilation of WIMP DM into $e^{+} e^{-}$final states for $m_{\chi} \lesssim 150 \mathrm{GeV}$, see Figs. 12. For light DM, down to $m_{\chi} \approx 10 \mathrm{MeV}$, new constraints on the $s$ - and $p$-wave annihilation based on Voyager-1 data, were presented in Ref. [31], as well as constraints on light PBHs, see Fig. 11.

As discussed in Ref. [22], searches for DM annihilation into neutrinos with the upcoming ARCA and ORCA instruments of the KM3NeT neutrino detector will significantly (by at least an order of magnitude) improve on existing searches, see Fig. 10. Among non-WIMP searches for DM, a highlight were new constraints on the evaporation of black holes (with mases around $10^{17} \mathrm{~s}$ to $10^{21} \mathrm{~s}$ ), which were presented in Ref. [14], based on transient searches with the HAWC observatory. These limits surpassed existing constraints by a factor of 5-10, see Fig. 7. Among various direct searches for WIMP-DM, results from the FUNK experiment for hidden photon DM searches for $m_{\bar{\gamma}}=1.94 \mathrm{eV}-8.40 \mathrm{eV}$ were presented in Ref. [41], see Fig. 15. Finally, first prototype studies for searches for the meteorite-like emission that would be expected from nuclearite DM were presented in Refs. [19, 20], with promising prospects for the proposed JEM-EUSO detector.

Acknowledgements. I would like to thank the organizers of the ICRC 2019 for a stimulating conference, and many of the contributors to the DM sessions for enlightening discussions. This research is funded by NWO through the VIDI research program "Probing the Genesis of Dark Matter" (680-47-532). 


\section{References}

[1] K. Bechtol, Searching for ultra-faint galaxies in three years of data from the Dark Energy Survey, in 36th International Cosmic Ray Conference (ICRC2019), vol. 36 of International Cosmic Ray Conference, p. 508, Jul, 2019.

[2] L. Oakes, Combined Dark Matter Searches Towards Dwarf Spheroidal Galaxies with Fermi-LAT, HAWC, HESS, MAGIC and VERITAS, in 36th International Cosmic Ray Conference (ICRC2019), vol. 36 of International Cosmic Ray Conference, p. 539, Jul, 2019.

[3] J. Lundeen and J. P. Harding, Dark Matter Searches with HAWC, in 36th International Cosmic Ray Conference (ICRC2019), vol. 36 of International Cosmic Ray Conference, p. 528, Jul, 2019.

[4] A. Albert, B. Anderson, K. Bechtol, A. Drlica-Wagner, M. Meyer, M. Sánchez-Conde et al., Searching for Dark Matter Annihilation in Recently Discovered Milky Way Satellites with Fermi-Lat, The Astrophysical Journal 834 (Jan, 2017) 110, [1611.03184].

[5] L. Rinchiuso, E. Moulin, C. Armand and V. Poireau, Dark Matter Search with H.E.S.S. Towards Ultra-faint Dwarf Nearby DES Satellites of the Milky Way, in 36th International Cosmic Ray Conference (ICRC2019), vol. 36 of International Cosmic Ray Conference, p. 542, Jul, 2019, 1908.04311.

[6] S. Hernández Cadena, V. Gammaldi, J. Serna Franco, R. Alfaro Molina, E. Karukes and P. Salucci, Constraints on cross section and lifetime of dark matter with HAWC Observations of dwarf Irregular galaxies, in 36th International Cosmic Ray Conference (ICRC2019), vol. 36 of International Cosmic Ray Conference, p. 520, Jul, 2019.

[7] C. Armand, V. Poireau, E. Moulin and L. Rinchiuso, Search for Dark Matter Signals Toward the Irregular Dwarf Galaxy WLM with H.E.S.S., in 36th International Cosmic Ray Conference (ICRC2019), vol. 36 of International Cosmic Ray Conference, p. 505, Jul, 2019.

[8] X. Bi, S. J. Lin and P. F. Yin, Perspective on dark matter annihilation limits from the LHAASO gamma-ray observation of dwarf Spheroidal galaxies, in 36th International Cosmic Ray Conference (ICRC2019), vol. 36 of International Cosmic Ray Conference, p. 509, Jul, 2019.

[9] D. Glawion, D. Malyshev, E. Moulin, L. Oakes, L. Rinchiuso, A. Viana et al., Unidentified Fermi Objects in the view of H.E.S.S. - Possible Dark Matter Clumps, in 36th International Cosmic Ray Conference (ICRC2019), vol. 36 of International Cosmic Ray Conference, p. 518, Jul, 2019.

[10] L. Rinchiuso, N. L. Rodd, E. Moulin and T. Slatyer, Hunting for Heavy Dark Matter in the Galactic Center with Ground-based Cherenkov Telescopes, in 36th International Cosmic Ray Conference (ICRC2019), vol. 36 of International Cosmic Ray Conference, p. 543, Jul, 2019, 1908.04317.

[11] D. Serini, F. Loparco and M. N. Mazziotta, Constraints on dark matter scattering with long-lived mediators using gamma-rays from the Sun, in 36th International Cosmic Ray Conference (ICRC2019), vol. 36 of International Cosmic Ray Conference, p. 544, Jul, 2019.

[12] D. Ninci, T. Inada, J. Rico, D. Kerszberg, M. Doro, S. Lombardi et al., Searching for Dark Matter decay signals in the Galactic halo with the MAGIC telescopes, in 36th International Cosmic Ray Conference (ICRC2019), vol. 36 of International Cosmic Ray Conference, p. 538, Jul, 2019.

[13] J. Lundeen, A Search For Dark Matter Signals in the Galactic Halo with HAWC, in 36th International Cosmic Ray Conference (ICRC2019), vol. 36 of International Cosmic Ray Conference, p. 529, Jul, 2019. 
[14] K. Engel, A. Peisker, P. Harding, J. Wood, I. Martinez-Castellanos, A. Albert et al., Setting Upper Limits on the Local Burst Rate Density of Primordial Black Holes Using HAWC, in 36th International Cosmic Ray Conference (ICRC2019), vol. 36 of International Cosmic Ray Conference, p. 516, Jul, 2019.

[15] V. Takhistov, New Venues in Formation and Detection of Primordial Black Hole Dark Matter, in 36th International Cosmic Ray Conference (ICRC2019), vol. 36 of International Cosmic Ray Conference, p. 546, Jul, 2019, 1908.01464.

[16] A. Archer and J. Buckley, ALP-photon Mixing in Pulsar Magnetospheres, in 36th International Cosmic Ray Conference (ICRC2019), vol. 36 of International Cosmic Ray Conference, p. 504, Jul, 2019.

[17] J. Majumdar, Spectral modulation of Galactic pulsars in the realm of photon-ALPs mixing., in 36th International Cosmic Ray Conference (ICRC2019), vol. 36 of International Cosmic Ray Conference, p. 530, Jul, 2019.

[18] F. Kajino, I. Ide, R. Ide, Y. Tameda, K. Shinozaki, M. E. Bertaina et al., Study for Moving Nuclearites and Interstellar Meteoroids using High Sensitivity CMOS Camera, in 36th International Cosmic Ray Conference (ICRC2019), vol. 36 of International Cosmic Ray Conference, p. 525, Jul, 2019.

[19] K. Shinozaki, A. Montanaro, M. E. Bertaina, F. Fenu and S. Ferrarese, Search for nuclearites by the satellite-based TUS air fluorescence detector, in 36th International Cosmic Ray Conference (ICRC2019), vol. 36 of International Cosmic Ray Conference, p. 545, Jul, 2019.

[20] L. Piotrowski, Limits on the flux of heavy stable macro particles from the Pi of the Sky project, in 36th International Cosmic Ray Conference (ICRC2019), vol. 36 of International Cosmic Ray Conference, p. 540, Jul, 2019.

[21] N. Iovine, J. A. Aguilar Sánchez, S. Baur, S. R. Gozzini and J. d. D. Zornoza Gómez, Combined Search for Neutrinos from Dark Matter Annihilation in the Galactic Centre using ANTARES and IceCube, in 36th International Cosmic Ray Conference (ICRC2019), vol. 36 of International Cosmic Ray Conference, p. 522, Jul, 2019, 1908.07300.

[22] S. R. Gozzini and J. d. D. Zornoza Gómez, Searches for dark matter with the ANTARES and KM3NeT neutrino telescopes, in 36th International Cosmic Ray Conference (ICRC2019), vol. 36 of International Cosmic Ray Conference, p. 552, Jul, 2019.

[23] S. Baur, Dark Matter Searches with the IceCube Upgrade, in 36th International Cosmic Ray Conference (ICRC2019), vol. 36 of International Cosmic Ray Conference, p. 506, Jul, 2019, 1908.08236.

[24] S. Navas, D. López-Coto, J. D. Zornoza and KM3NeT Collaboration, Dark Matter Searches from the Sun with the KM3NeT-ORCA detector, in 36th International Cosmic Ray Conference (ICRC2019), vol. 36 of International Cosmic Ray Conference, p. 536, Jul, 2019.

[25] A. Kheirandish, C. Arguelles, J. Lazar and Q. Liu, Search for Dark Matter Annihilation to Neutrinos from the Sun, in 36th International Cosmic Ray Conference (ICRC2019), vol. 36 of International Cosmic Ray Conference, p. 527, Jul, 2019.

[26] G. Renzi, Search for dark matter annihilation in the center of the Earth with 8 years of IceCube data, in 36th International Cosmic Ray Conference (ICRC2019), vol. 36 of International Cosmic Ray Conference, p. 541, Jul, 2019, 1908.07255. 
[27] C. Tönnis, The search for dark matter with metastable mediators with the IceCube observatory, in 36th International Cosmic Ray Conference (ICRC2019), vol. 36 of International Cosmic Ray Conference, p. 548, Jul, 2019, 1908.07243.

[28] S. R. Gozzini, C. Lagunas, F. Sala and J. d. D. Zornoza Gómez, Sensitivity of the ANTARES neutrino telescope for secluded dark matter searches, in 36th International Cosmic Ray Conference (ICRC2019), vol. 36 of International Cosmic Ray Conference, p. 519, Jul, 2019.

[29] M. Chianese, Potential Dark Matter Signals at Neutrino Telescopes, in 36th International Cosmic Ray Conference (ICRC2019), vol. 36 of International Cosmic Ray Conference, p. 514, Jul, 2019.

[30] X. Bi, Two-zone Diffusion of Electrons and Positrons from Geminga Explains the Positron Anomaly, in 36th International Cosmic Ray Conference (ICRC2019), vol. 36 of International Cosmic Ray Conference, p. 510, Jul, 2019.

[31] M. Boudaud, Voyager Probing Dark Matter, in 36th International Cosmic Ray Conference (ICRC2019), vol. 36 of International Cosmic Ray Conference, p. 512, Jul, 2019.

[32] M. N. Mazziotta, A. Cuoco, P. De La Torre Luque, F. Loparco and D. Serini, Search for dark matter signatures in the cosmic-ray electron and positron spectrum measured by the Fermi Large Area Telescope, in 36th International Cosmic Ray Conference (ICRC2019), vol. 36 of International Cosmic Ray Conference, p. 531, Jul, 2019.

[33] H. Motz, Y. Asaoka and S. Bhattacharyya, Interpretation of the CALET Electron+Positron Spectrum concerning Dark Matter Signatures, in 36th International Cosmic Ray Conference (ICRC2019), vol. 36 of International Cosmic Ray Conference, p. 533, Jul, 2019.

[34] P. von Doetinchem, Status of cosmic-ray antinuclei searches, in 36th International Cosmic Ray Conference (ICRC2019), vol. 36 of International Cosmic Ray Conference, p. 550, Jul, 2019.

[35] K. C. Y. Ng, Reverse Direct Detection: Cosmic Ray Scattering With Light Dark Matter, in 36th International Cosmic Ray Conference (ICRC2019), vol. 36 of International Cosmic Ray Conference, p. 537, Jul, 2019.

[36] M. Boudaud, Y. Genolini, L. Derome, J. Lavalle, D. Maurin, P. Salati et al., AMS-02 Antiprotons are Consistent with a Secondary Astrophysical Origin, in 36th International Cosmic Ray Conference (ICRC2019), vol. 36 of International Cosmic Ray Conference, p. 38, Jul, 2019, 1906.07119.

[37] R. Munini, M. Boezio, P. von Doetinchem, N. Marcelli, A. Stoessl and E. Vannuccini, Event reconstruction performance with the GAPS experiment, in 36th International Cosmic Ray Conference (ICRC2019), vol. 36 of International Cosmic Ray Conference, p. 535, Jul, 2019.

[38] K. Jędrzejczak, Z. Debicki, M. Kasztelan, W. Marszal, J. Orzechowski, M. Przybylak et al., The BSUIN project, in 36th International Cosmic Ray Conference (ICRC2019), vol. 36 of International Cosmic Ray Conference, p. 523, Jul, 2019.

[39] J. Jo, Recent results from the DM-Ice17 and COSINE-100 experiment, in 36th International Cosmic Ray Conference (ICRC2019), vol. 36 of International Cosmic Ray Conference, p. 524, Jul, 2019.

[40] D. Baxter, Recent Results from the Dark Matter Search of DAMIC-100 at SNOLAB, in 36th International Cosmic Ray Conference (ICRC2019), vol. 36 of International Cosmic Ray Conference, p. 507, Jul, 2019.

[41] A. Andrianavalomahefa, K. Daumiller, R. Engel, B. Döbrich, J. Jaeckel, M. Kowalski et al., Search for dark photons as candidates for Dark Matter with FUNK, in 36th International Cosmic Ray Conference (ICRC2019), vol. 36 of International Cosmic Ray Conference, p. 517, Jul, 2019. 